Tohoku J. exp. Med., 1979, 129, 1-8

\title{
Biopsy under Direct Vision for the Diagnosis of Crohn's Disease
}

\author{
Hikaru Watanabe, Nobuo Hiwatashi and Yoshio Goto \\ The Third Department of Internal Medicine, Tohoku University \\ School of Medicine, Sendai 980
}

\begin{abstract}
Watanabe, H., Hiwatashi, N. and Goto, Y. Biopsy under Direct Vision for the Diagnosis of Crohn's Disease. Tohoku J. exp. Med., 1979, 129 (1), 1-8-In order to clarify the role of biopsy in distinguishing Crohn's disease from intestinal tuberculosis before surgery, the histologic findings of the biopsy specimens from 8 cases of Crohn's disease were investigated in comparison with those from 19 cases of intestinal tuberculosis. The samples were obtained under direct vision through a fibercolonoscope from areas showing a cobblestone appearance or narrowing, and then approximately 60 serial sections of each sample were prepared whenever Crohn's disease was suspected on the basis of $\mathrm{x}$-ray and endoscopic findings and clinical features. As a result, characteristic histologic findings such as small and discrete mucosal, noncaseating granulomas, disproportional inflammation, discontinuity of the lesion, and submucosal neuromatous hyperplasia were found in the majority of Crohn's disease cases. Granulomas were also found frequently in the cases of intestinal tuberculosis, although other findings were rare in such cases. Among granulomas in the intestinal tuberculosis cases, the number of submucosal granulomas was large and they showed a tendency to confluence, but the granulomas in the mucosa were small and discrete, as was the cases in the granulomas in Crohn's disease. There were no cases of caseating granulomas in the intestinal tuberculosis cases and the results of cultures for tubercle bacilli from biopsied samples were negative in the majority of cases in which cultures were attempted. Accordingly, it is thought to be difficult to differentiate Crohn's disease correctly from intestinal tuberculosis on biopsy alone, although biopsy under direct vision itself is a useful diagnostic procedure in Crohn's disease. Crohn's disease; intestinal tuberculosis; fibercolonoscope
\end{abstract}

With the development of a fibercolonoscope by us in 1969, observation and study of the deeper portion of the large intestine have become relatively easy and have allowed for biopsy under direct vision in many cases of Crohn's disease with deep colon lesions (Watanabe et al. 1975a, b). We report here an investigation on the differential diagnosis of Crohn's disease from intestinal tuberculosis.

\section{Materials and Methods}

Materials consisted of 13 cases diagnosed as Crohn's disease and 24 cases found to be intestinal tuberculosis at resection or by therapeutic differential diagnosis. All of 37

Received for publication, September 20, 1978.

This paper was presented at the 19th Autumnal Meeting of the Japanese Society of Gastroenterology held in Nara on 18-20 October, 1977 and at the lst World Congress of Colo-Proctology held in Madrid on 1-3 June, 1978. 
cases were detected at the Third Department of Internal Medicine, Tohoku University School of Medicine during a 20-year-period from 1958 until 1977. A diagnosis of Crohn's disease was made based on the criteria of the Japanese Research Committee for Crohn's Disease of the Japanese Society of Gastroenterology (1976), which are shown in Table 1, excluding the following diseases: intestinal tuberculosis, ulcerative colitis, ischemic (entero)colitis, radiation (entero)colitis, intestinal Behçet's disease, simple (nonspecific) ulcer of the small or large intestine, "nonspecific multiple ulcers of the small intestine", acute terminal ileitis, etc. With regard to the diagnosis of intestinal tuberoulosis, a caseating granuloma was demonstrated in resected tissue in 3 cases and tubercle bacilli were demonstrated by culture of biopsied tissue from the lesion in one case. In 4 cases where there was no caseating granuloma in the resected sample, the diagnosis was based on both macroscopic and histologic findings of the resected samples, such as the presence of noncaseating granulomas with confluent tendency and the lack of fissuring and edema microscopically as well as the presence of transverse ulcerations with scarring macroscopically, as described by Tandon and Prakash (1972). In another 16 cases, which were treated non-surgically, the diagnosis was made on the basis of both the demonstration of noneaseating granulomas in biopsied tissue and therapeutic differential diagnosis with anti-tubercular therapy, in addition to the clinical features, x-ray findings and endoscopic findings. Furthermore, the tuberculin test was negative or doubtfully positive in 7 of 11 cases of Crohn's disease tested, but positive in 17 of 19 cases of intestinal tuberculosis tested. The tubercle bacilli from sputum were positive in 3 of 16 cases of intestinal tuberculosis tested.

Among these 37 cases, biopsy under direct vision was performed in 8 cases of Crohn's disease and 19 cases of intestinal tuberculosis. With regard to the histologic findings of the biopsy specimens, approximately 60 serial sections per specimen at $4 \mu \mathrm{m}$ intervals were obtained using a revolving microtome, a total of 300 to 1,000 serial sections per case were obtained and characteristic findings and their rates of appearance were studied. The samples were collected with punch forceps from areas showing a cobblestone appearance or narrowing in major lesions. Samples collected from aphthoid ulcers or from apparently normal mucosa located between major lesions were not included in the examination. Furthermore, histologic examination was performed on resected samples of both types of lesion and the results were used as supporting evidence.

\section{Results}

Discrete noncaseating epitheloid cell granulomas were found in 6 of the 8 cases of Crohn's disease, among which 5 were in the mucosa and one was in the submucosa (Table 2, Figs. 1 and 2). One of the 7 granulomas found in the mucosa in the 5 cases was found near a capillary (Fig. 3). Although the mucosa appeared nearly normal, intense inflammation in the submucosa, so-called disproportional inflammation, was seen in 4 of 6 examined cases (Table 2, Fig. 4). Discontinuity of the lesion, in which normal mucosa is found between lesions, was seen in 7 of 8 cases and neuromatous hyperplasia in the submucosa was seen in all 7 examined cases (Table 2, Fig. 4). In contrast, of the 18 cases of intestinal tuberculosis 15 showed granulomas, 8 of which were in the mucosa and 10 of which were in the submucosa (Table 2). Among these, the number of submucosal granulomas was large and they showed a tendency to confluence, but the granulomas in the mucosa were small and discrete, as was the case in the granulomas in Crohn's disease (Figs. 5, 6 and 7). There were no cases of caseating granulomas in the intestinal tuberculosis cases and the results of cultures for tubercle bacilli from the biopsied samples were negative in 8 of 9 cases where cultures were attempted. In addition, disproportional inflammation, discontinuity of the 


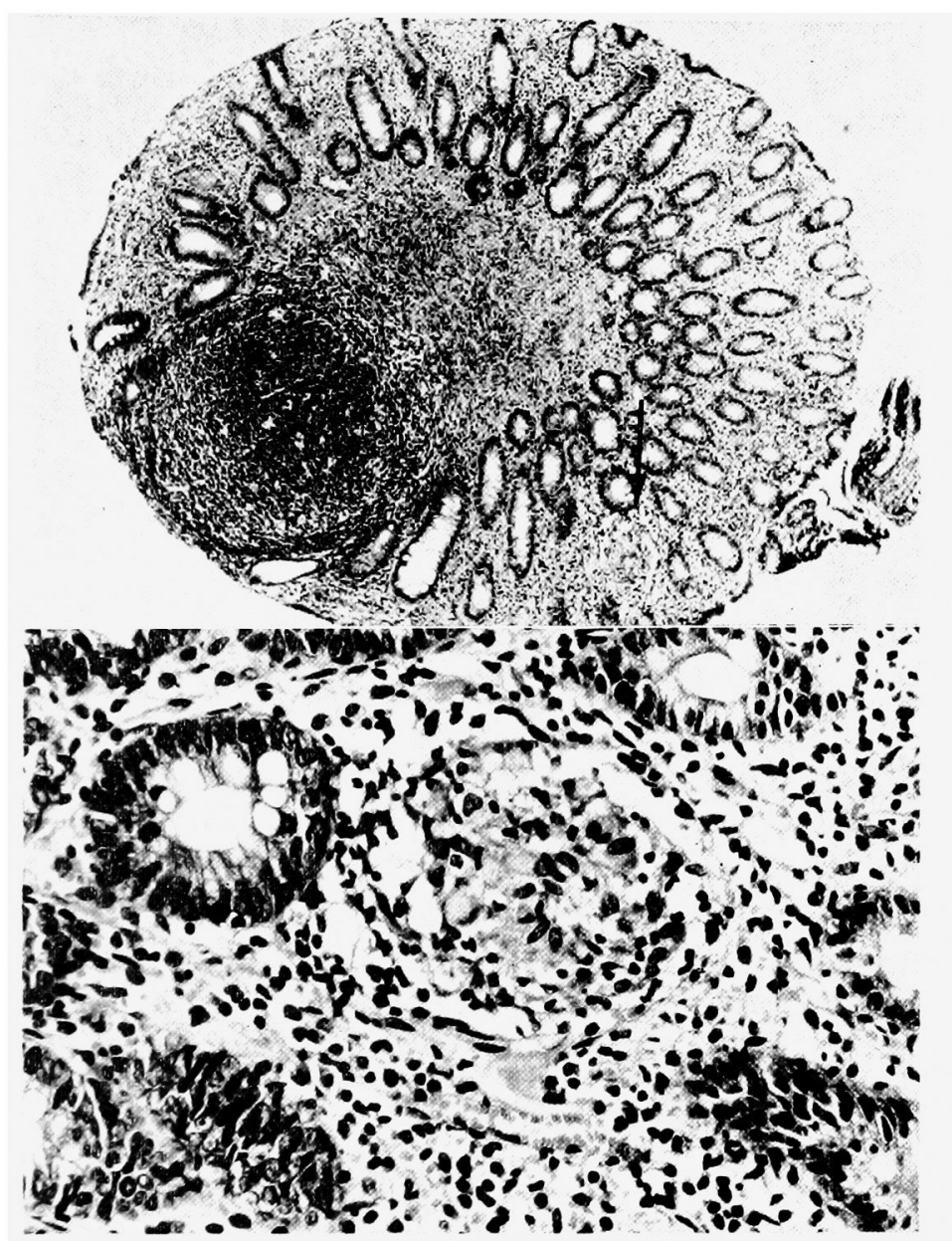

Fig. 1 (upper). Histologic findings of biopsy specimen in a case of Crohn's disease, showing a small and discrete noncaseating granuloma in the mucosa $(\rightarrow) . \times 90$.

Fig. 2 (lower). Magnification of Fig. $1 . \times 360$.

TABLE 1. The diagnostic criteria of the Japanese Research Committee for Crohn's Disease

\begin{tabular}{lccccc}
\hline & Examination & x-Ray & Endoscopy & Biopsy & Specimen \\
\hline $\begin{array}{l}\text { Discontinuity of lesion } \\
\text { Cobblestone appearance }\end{array}$ & & + & + & + & + \\
$\quad \begin{array}{l}\text { and/or longitudinal ulcer } \\
\text { Transmural inflammation }\end{array}$ & + (tumor) & + (stenosis) & + (stenosis) & & + \\
$\begin{array}{l}\text { Granuloma } \\
\text { Fissures and/or fistula(e) }\end{array}$ & $+^{\prime}$ & + & & $+^{\prime \prime}$ & $+^{\prime \prime}$ \\
Anal lesion & $+^{\prime}$ & $+^{\prime}$ & & & $+^{\prime}$ \\
\end{tabular}

Crohn's disease $=+++$ and $t^{\prime}$; or ++ and + " 


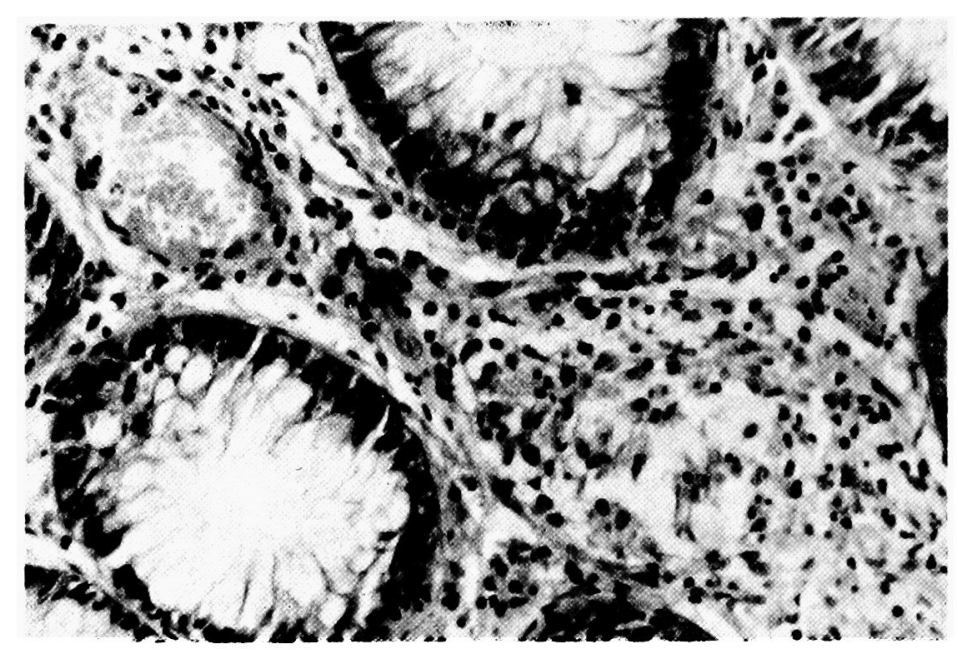

Fig. 3. Histologic findings of biopsy specimen, in another case of Crohn's disease, showing a noncaseating granuloma in the mucosa. The granuloma is noted near a capillary. $\times 360$.

TABLE 2. Microscopic differences in biopsy of Crohn's disease and intestinal tuberculosis

\begin{tabular}{llc}
\hline & Crohn's disease & $\begin{array}{c}\text { Intestinal } \\
\text { tuberculosis }\end{array}$ \\
\hline Mucosal inflammation & 6 & $15 / 18$ \\
Goblet cell depeltion & 4 & $12 / 18$ \\
Crypt abscesses & 3 & $7 / 18$ \\
Erosions & 5 & $2 / 18$ \\
Ulcers & 1 & $1 / 18$ \\
Granulation tissue & 4 & 11 \\
Disarrangement of the glands & 8 & $17 / 18$ \\
Regeneration of the glands & 8 & $18 / 18$ \\
Paneth cell metaplasia & 3 & $16 / 18$ \\
Granuloma(s) & 6 & $15 / 18$ \\
Disproportional inflammation & $4 / 6$ & $5 / 18$ \\
Discontinuity of the lesion & 7 & $7 / 18$ \\
Neuromatous hyperplasia & $7 / 7$ & $7 / 18$ \\
Total number of cases & & 19 \\
\hline
\end{tabular}

lesion, and neuromatous hyperplasia were noted in 5, 7, and 7 cases, respectively, among the 18 cases of intestinal tuberculosis (Table 2). Furthermore, no striking differences in histologic findings of the biopsy specimens were noted between 3 cases of definite intestinal tuberculosis diagnosed on the basis of the demonstration of caseating granulomas or tubercle bacilli and 16 other cases of probable intestinal tuberculosis without these two findings.

For support of the biopsy findings, a comparison of the histologic findings of the resected samples in Crohn's disease and intestinal tuberculosis was made. This showed that fissuring ulcer, lymphangiectasia, disproportional inflammation, 


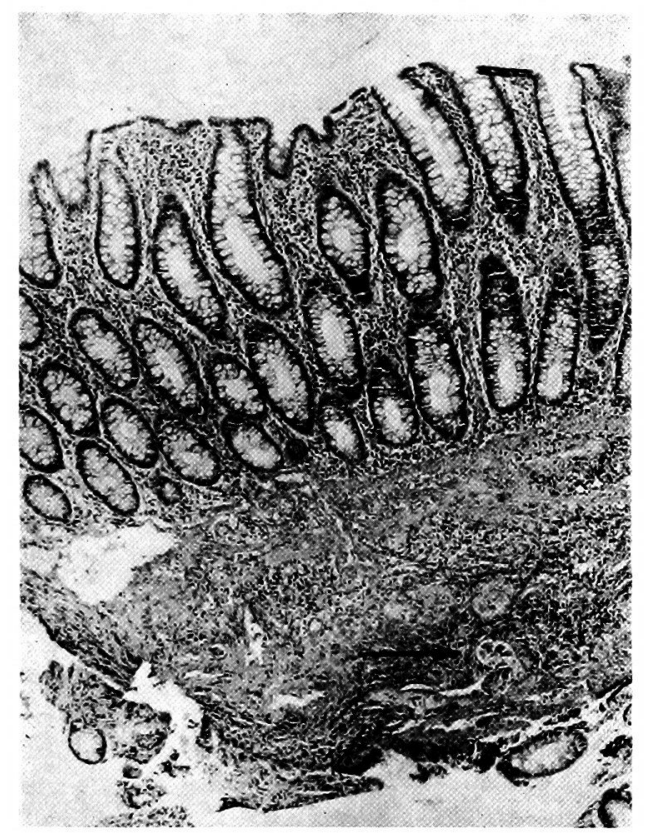

Fig. 4. Histologic findings of biopsy specimen in a case of Crohn's disease, showing disproportional inflammation and neuromatous hyperplasia in the submucosa $(\rightarrow)$. $\times 90$.

TABLE 3. Microscopic differences in resected samples of Crohn's disease and intestinal tuberculosis

\begin{tabular}{lcc}
\hline & Crohn's disease & $\begin{array}{c}\text { Intestinal } \\
\text { tuberculosis }\end{array}$ \\
\hline Transmural inflammation & 9 & 7 \\
Granuloma(s) & 9 & 7 \\
Fissuring ulcer(s) & 7 & 0 \\
Lymphangiectasia & 6 & 0 \\
Disproportional inflammation & 9 & 1 \\
Discontinuity of the lesion & 8 & 0 \\
Neuromatous hyperplasia & 7 & 1 \\
Total number of cases & 9 & 7 \\
\hline
\end{tabular}

discontinuity of the lesion and submucosal neuromatous hyperplasia were frequent in Crohn's disease, whereas those findings were almost never found in intestinal tuberculosis (Table 3). With regard to granulomas, they were found in all 9 of the Crohn's disease cases and all 7 of the intestinal tuberculosis, and caseation was seen in 3 of the latter (Table 3). There were no notable differences in the histologic findings besides granulomas between 3 definite cases with caseating granulomas and 4 probable cases without them. 


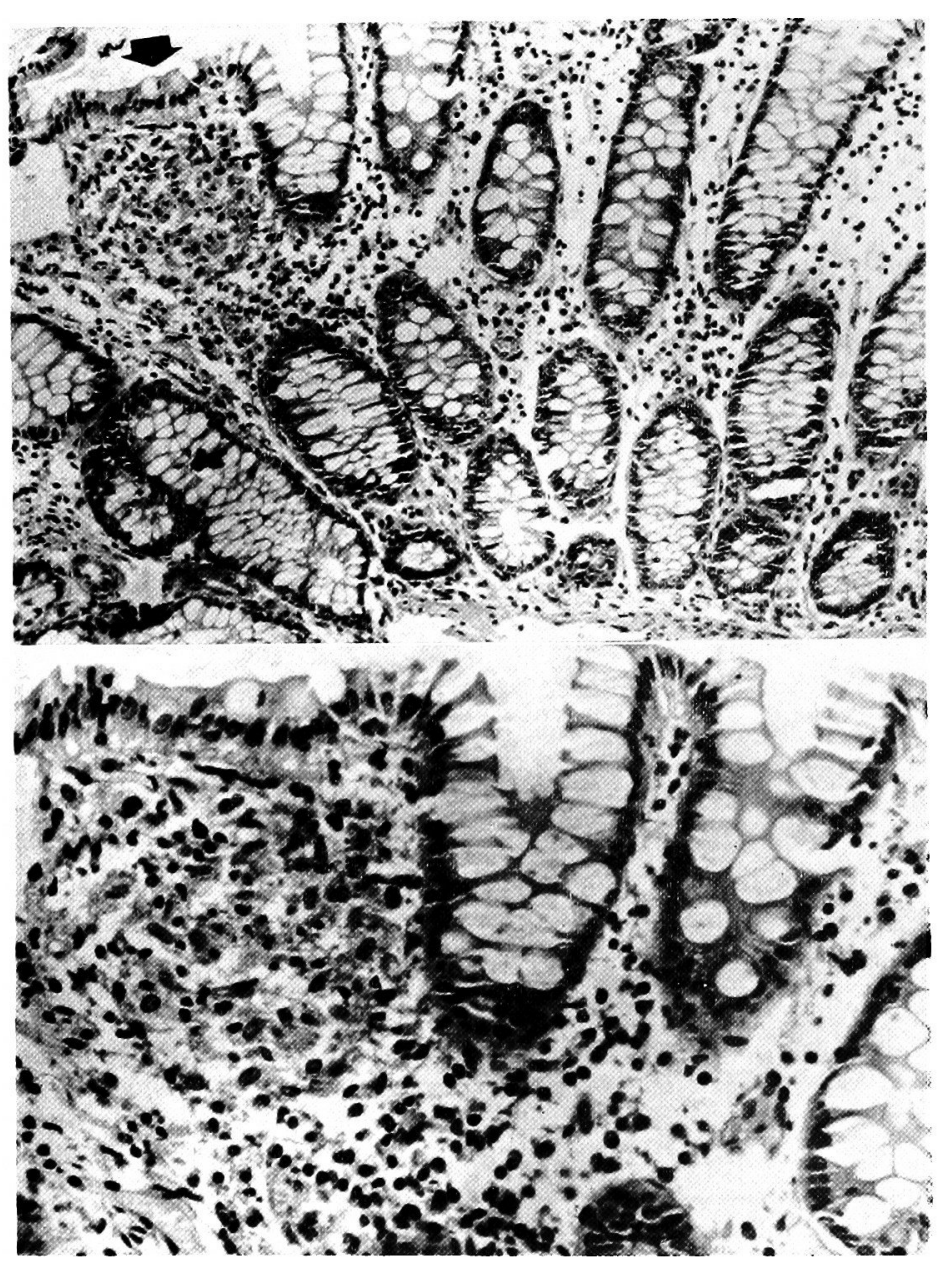

Fig. 5. Histologic findings of biopsy specimen in a case of intestinal tuberculosis. A small and isolated, noncaseating granuloma is observed in the mucosa $(\rightarrow) . \times 180$.

Fig. 6. Magnification of Fig. 5. $\times 360$.

\section{Discussion}

In order to clarify the role of biopsy in distinguishing Crohn's disease from intestinal tuberculosis before surgery, the histologic findings of the biopsy specimens from 8 cases of Crohn's disease with large intestinal lesions were investigated in comparison with those from 19 cases of intestinal tuberculosis. By collecting with punch forceps samples which include a sufficient amount of submucosa and examining them by serial sections, a high incidence of granulomas were detected in Crohn's disease and intestinal tuberculosis. For the differential diagnosis of these two diseases, it is necessary to check for characteristic histologic findings, namely, disproportional inflammation, discontinuity of the lesion, and submucosal neuromatous hyperplasia, and diagnosis must be made considering all 


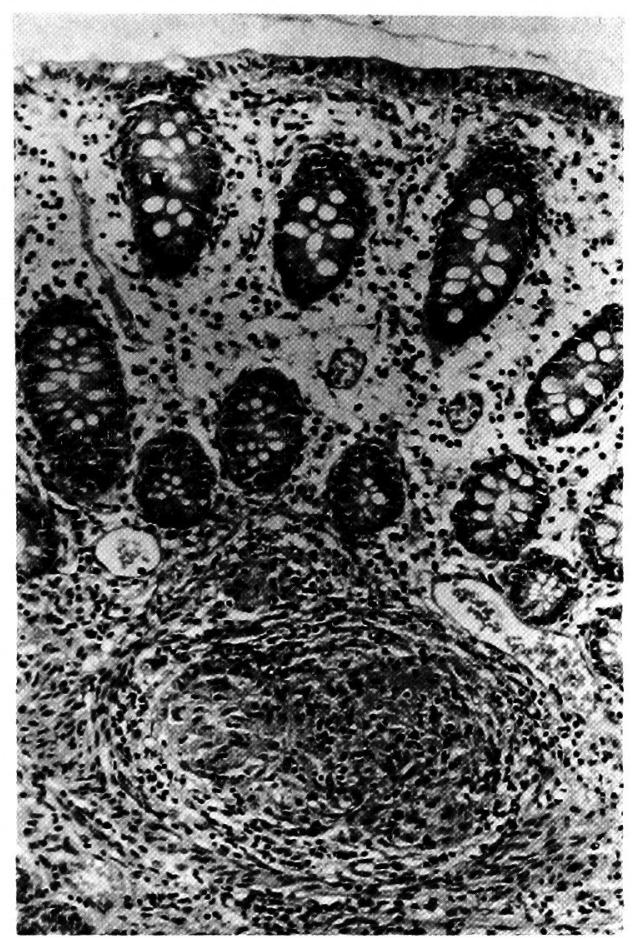

Fig. 7. Histologic findings of biopsy specimen in the same case as shown in Figs. 5 and 6 , showing large and confluent granulomas in the submucosa. $\times 180$.

of these factors. However, tubercle bacilli could be detected only rarely and caseating granulomas could not be proven by biopsy, and it was difficult to distinguish between the small and discrete mucosal, noncaseating granulomas in Crohn's disease and those in intestinal tuberculosis. It is, therefore, thought that in cases where the diagnosis is inconclusive it is absolutely necessary to follow the clinical course of the disease while observing the effects of anti-tubercular drugs. In other words, biopsy under direct vision through an endoscope is a useful diagnostic procedure in Crohn's disease, but it is nonetheless still thought to be difficult to differentiate it correctly from intestinal tuberculosis on biopsy alone.

Furthermore, it appears that there is no report in the literature concerning biopsy of the lesions in Crohn's disease except ours (Watanabe et al. 1975a).

\section{References}

1) Japanese Research Committee for Crohn's Disease (Chairman: Yamagata, S.) (1976) Diagnostic criteria for Crohn's disease. Jap. J. Gastroent., 73, 1467-1478. (Japanese)

2) Tandon, H.D. \& Prakash, A. (1972) Pathology of intestinal tuberculosis and its distinction from Crohn's disease. Gut, 13, 260-269.

3) Watanabe, H., Narasaka, T., Imai, H., Uezu, J.M., Numazawa, M., Shoji, K., Ishikawa, M. \& Yamagata, S. (1975a) Fibercolonoscopy for the diagnosis of granulomatous colitis. Tohoku J. exp. Med., 115, 285-296. 
4) Watanabe, H., Narasaka, T. \& Yamagata, S. (1975b) Studies on the fibercolonoscopy — with special reference to the latest procedures. Toholku J. exp. Med., 117, 385-396. 